TRANSACTIONS OF THE

AMERICAN MATHEMATICAL SOCIETY

Volume 355 , Number 12, Pages 4865-4880

S 0002-9947(03)03219-7

Article electronically published on July 28, 2003

\title{
THE LIMITING CURVE OF JARNÍK'S POLYGONS
}

\author{
GREG MARTIN
}

\begin{abstract}
In 1925, Jarník defined a sequence of convex polygons for use in constructing curves containing many lattice points relative to their curvatures. Properly scaled, these polygons converge to a certain limiting curve. In this paper we identify this limiting curve precisely, showing that it consists piecewise of arcs of parabolas, and we discuss the analogous problem for sequences of polygons arising from generalizations of Jarník's construction.
\end{abstract}

\section{INTRODUCTION}

In 1925, Jarník 3] defined a sequence of convex polygons for use in constructing curves containing many lattice points relative to their curvature. Given a positive integer $Q$, let $V_{Q}$ denote the set of all primitive integral vectors in the square of side length $2 Q$ centered at the origin; that is,

$$
V_{Q}=\left\{(q, a) \in \mathbb{Z}^{2}: \operatorname{gcd}(q, a)=1, \max \{|a|,|q|\} \leq Q\right\} .
$$

Then the Jarník polygon $P_{Q}$ is the unique (up to translation) convex polygon whose sides are precisely the vectors in $V_{Q}$. In other words, $P_{Q}$ is the polygon whose vertices can be obtained by starting from an arbitrary point in $\mathbb{R}^{2}$ and adding the vectors in $V_{Q}$ one by one, traversing those vectors in a counterclockwise direction. For example, the forty-eight vectors in $V_{4}$, listed in counterclockwise order, are

$$
\begin{aligned}
\ldots,(1,0),(4,1), & (3,1),(2,1),(3,2),(4,3),(1,1), \\
& (3,4),(2,3),(1,2),(1,3),(1,4),(0,1),(-1,4), \ldots,
\end{aligned}
$$

and hence $P_{4}$ is the tetracontakaioctagon that can be translated to have vertices at

$$
\begin{aligned}
\ldots,(-1,0),(0,0),(4,1),(7,2),(9,3),(12,5),(16,8),(17,9), \\
(20,13),(22,16),(23,18),(24,21),(25,25),(25,26),(24,30), \ldots
\end{aligned}
$$

These polygons were featured on a recent cover of the Notices of the American Mathematical Society in connection with an article of Iosevich [2], and are discussed in further detail in [1, Chapter 2]. Figure[1 shows the four sets of vectors $V_{1}$ through $V_{4}$ and the four polygons $P_{1}$ through $P_{4}$ which they generate. $]$

The polygons $P_{Q}$ have the same eight-fold dihedral symmetry as the unit square $E=[-1,1]^{2}$ and, if properly scaled and translated, can be made to pass through the points $( \pm 1,0)$ and $(0, \pm 1)$. We denote by $\tilde{P}_{Q}$ these scaled and translated copies of $P_{Q}$. In a coda to [2, Casselman suggests, based on empirical evidence, "that

Received by the editors June 20, 2002.

2000 Mathematics Subject Classification. Primary 52C05; Secondary 11H06.

${ }^{1}$ Figure 1 and the boxed portion of Figure 2 are a modification of the cover image for the June/July 2001 issue of the Notices of the AMS ; they were drawn by Bill Casselman in Postscript. 


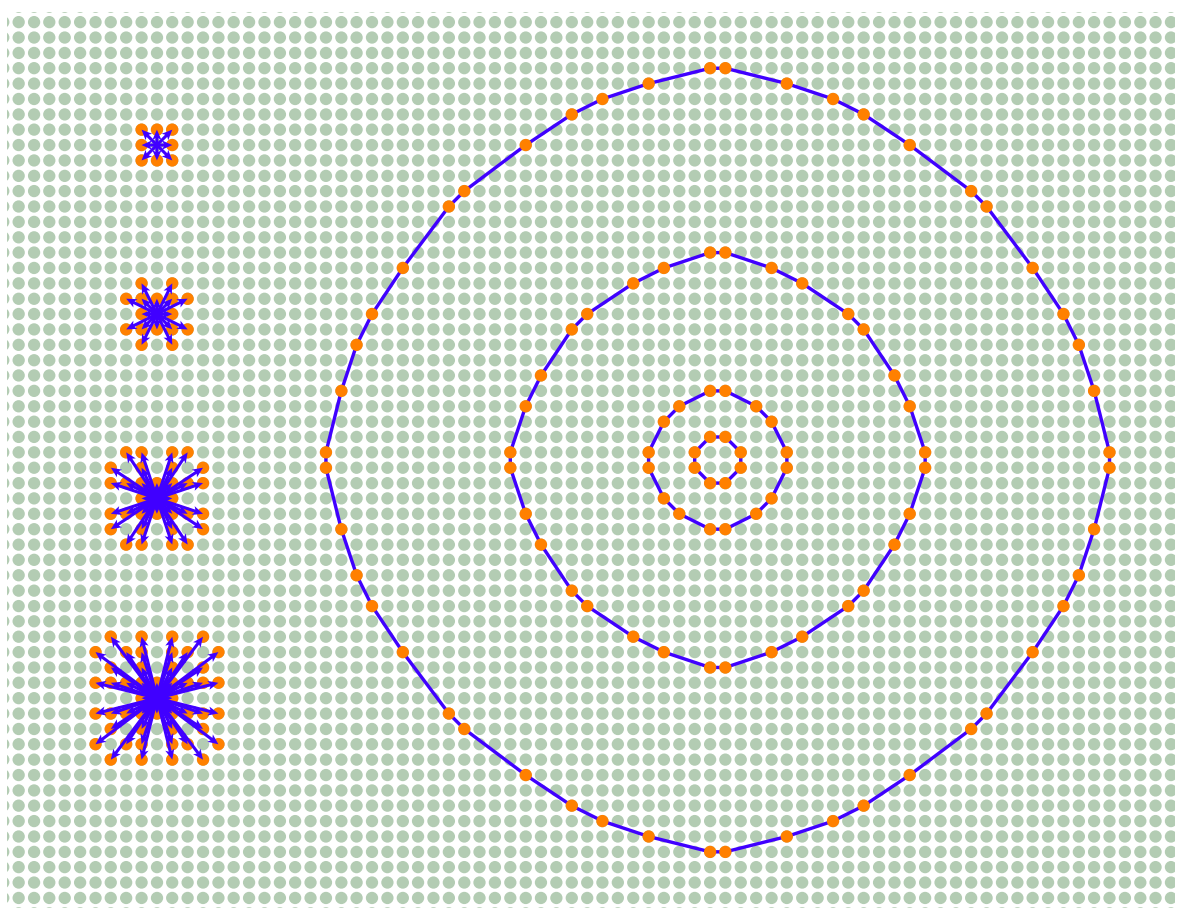

FiguRE 1. Jarník polygons, right, and their generating sets of vectors, left
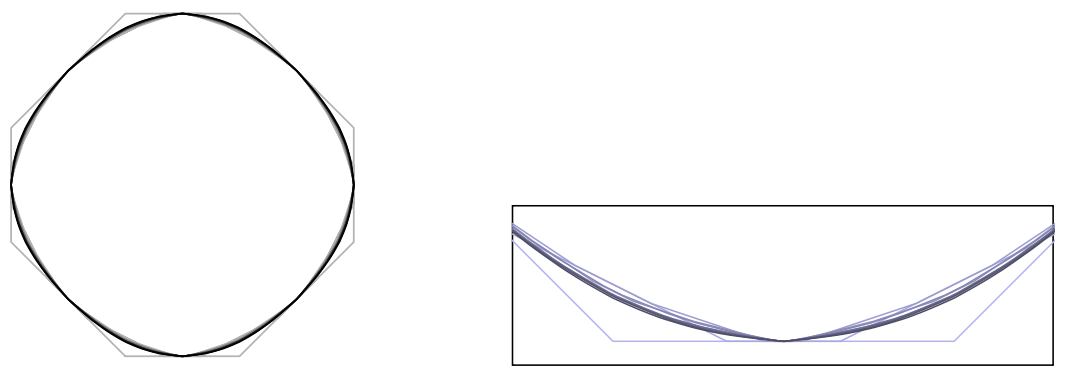

FiguRE 2. Scaled Jarník polygons superimposed

the scaled polygons $\left[\left\{\tilde{P}_{Q}\right\}\right]$ converge to a somewhat ragged limit curve". The first several scaled Jarník polygons have been superimposed in Figure2, with a magnified portion shown in the box to the right; the darker polygons correspond to larger values of $Q$. The purpose of this paper is to calculate explicitly the limiting curve of the Jarník polygons. Indeed, we present several variations on Jarník's polygons and calculate the corresponding limit curves, in many cases explicitly and in other cases parametrically.

If $A$ is a curve in $\mathbb{R}^{2}$ and $\varepsilon>0$, let $A(\varepsilon)$ denote the $\varepsilon$-neighborhood of $A$, that is, the set of all points whose distance to $A$ is less than $\varepsilon$. Given a sequence of curves $A_{1}, A_{2}, \ldots$, we say that the curves $\left\{A_{j}\right\}$ converge to $A$ if for every $\varepsilon>0$, there is 

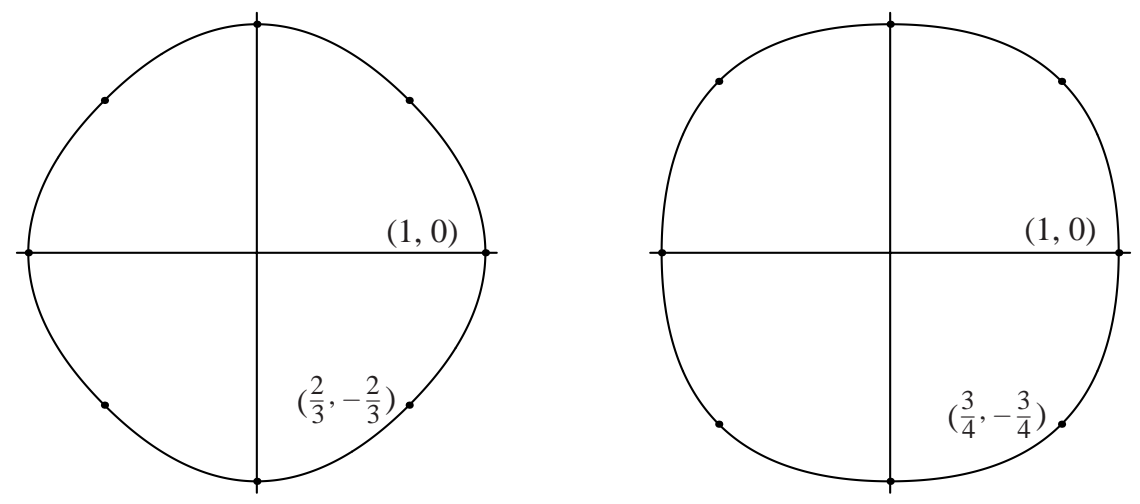

Figure 3 . The curves $C$, left, and $C_{1}$, right

some integer $j(\varepsilon)$ such that $A_{j}$ is contained in $A(\varepsilon)$ for every $j>j(\varepsilon)$. Our main result, which we prove in Section 2, is the following theorem.

Theorem 1. Let $C$ be the curve that contains the graph of the equation

$$
y=\frac{3}{4} x^{2}-1, \quad-\frac{2}{3} \leq x \leq \frac{2}{3},
$$

and that is invariant under rotation by $\pi / 2$ around the origin. Then the scaled Jarnik polygons $\left\{\tilde{P}_{Q}\right\}$ converge to $C$.

The curve $C$ is infinitely differentiable everywhere except at the four points $\left( \pm \frac{2}{3}, \pm \frac{2}{3}\right)$, where it is only twice differentiable. Although this limiting curve is surprisingly tame, it is the case that the local "curvatures" of the scaled Jarník polygons oscillate rather than tending to the corresponding local curvatures of $C$. We describe this phenomenon in Theorem 7 , the statement and proof of which appear in Section 6.

It is interesting to note the relationship between $C$ and the curve $C_{1}$, defined as the graph of the equation $\sqrt{1-|x|}+\sqrt{1-|y|}=1$ (these two curves are displayed in Figure 3). Indeed, if $C$ is rotated by $\frac{\pi}{4}$ and then expanded by the factor $\frac{3}{2 \sqrt{2}}$, so that the image again passes through the four points $( \pm 1,0)$ and $(0, \pm 1)$, then the resulting curve is none other than $C_{1}$. Vershik [5] showed that $C_{1}$ is the "generic" shape of a convex polygon with lattice point vertices, in the sense that if $P$ is chosen at random uniformly from among all convex polygons whose vertices lie in the set $\left(\frac{1}{Q} \mathbb{Z}\right)^{2} \cap E$, then, with probability approaching 1 as $Q$ tends to infinity, the polygon $P$ lies within any prescribed open neighborhood of $C_{1}$.

That the curves $C$ and $C_{1}$ differ only up to rotation and scaling suggests that rotating the domain demarcating the vectors in $V_{Q}$ might yield interesting results. Very generally, given a set $S \subset \mathbb{R}^{2}$, we may define the set of vectors

$$
V_{Q}(S)=\left\{(a, b) \in \mathbb{Z}^{2}: \operatorname{gcd}(a, b)=1,\left(\frac{a}{Q}, \frac{b}{Q}\right) \in S\right\} .
$$

We also define the corresponding convex polygons $P_{Q}(S)$ whose sides are precisely the vectors in $V_{Q}(S)$. If we take $S=E$, we recover the vectors $V_{Q}$ and polygons $P_{Q}$ in Jarník's original definition above. While these definitions make sense for any set $S$, it seems reasonable in practice to restrict ourselves to sets $S$ that are star-shaped with respect to the origin and that are the closures of their interiors; indeed, in this paper we will only consider such sets $S$ centered at the origin that 

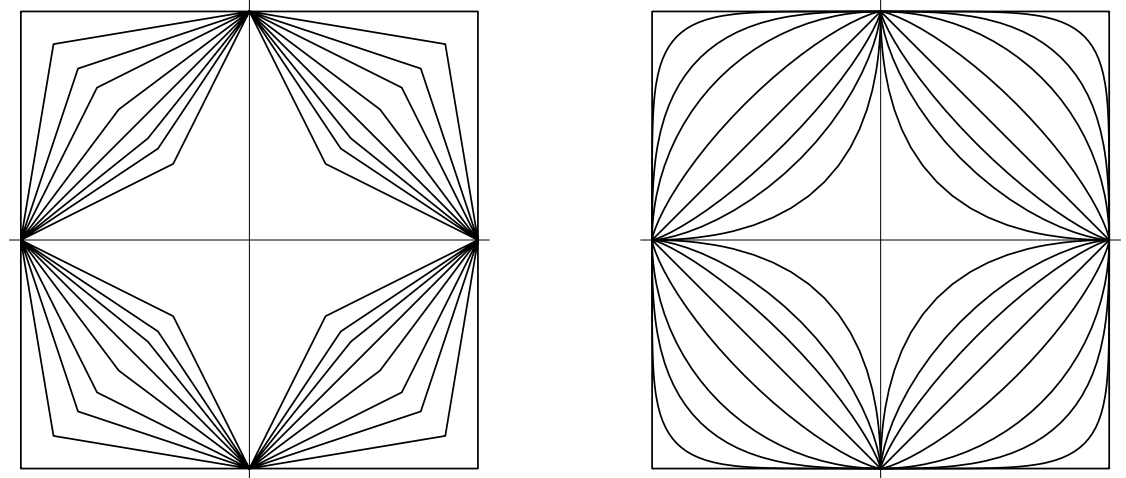

Figure 4 . The families $O_{\delta}$, left, and $B_{p}$, right

in addition have the same eight-fold dihedral symmetry as the unit square. We call the $P_{Q}(S)$ generalized Jarnik polygons. For such sets $S$, we define $\tilde{P}_{Q}(S)$ to be the scaled and translated copy of $P_{Q}(S)$ that passes through the four points $( \pm 1,0)$ and $(0, \pm 1)$.

Let $D$ denote the "unit diamond", namely the square with vertices $( \pm 1,0)$ and $(0, \pm 1)$. The relationship between $C$ and $C_{1}$ suggests the following theorem, which we establish in Section 3.

Theorem 2. The scaled generalized Jarnik polygons $\left\{\tilde{P}_{Q}(D)\right\}$ converge to $C_{1}$.

Put another way, the generalized Jarník polygons $P_{Q}(D)$ are "generic" in shape, in the sense of Vershik's theorem.

In this paper we also compute the limit curves corresponding to the $\tilde{P}_{Q}(S)$ for two families of sets $S$, both of which were chosen because they interpolate between the unit square $E$ and the unit diamond $D$.

- For any positive real number $\delta$, let $O_{\delta}$ be the octagon with vertices at $( \pm 1,0)$ and $(0, \pm 1)$ and the four points $\left( \pm \frac{\delta}{1+\delta}, \pm \frac{\delta}{1+\delta}\right)$. These octagons have eight-fold dihedral symmetry, and the slopes of the two edges meeting at the vertex $(1,0)$ are $\pm \delta$. When $\delta=1$ and as $\delta \rightarrow \infty$, the octagons $O_{\delta}$ degenerate to the squares $D$ and $E$, respectively. Figure 4 shows several of these octagons, with $O_{1 / 2}$ the innermost and $O_{\infty}$ the outermost. In Section 4 we calculate the limiting curves of the polygons $\left\{\tilde{P}_{Q}\left(O_{\delta}\right)\right\}$ explicitly, and it turns out that for all values of $\delta$ these limiting curves are comprised of pieces of parabolas.

- For any positive real number $p$, let $B_{p}$ be the set $\left\{|x|^{p}+|y|^{p} \leq 1\right\}$. When $p \geq 1$, the set $B_{p}$ is simply the closed unit ball in $\mathbb{R}^{2}$ under the $\ell^{p}$ metric. Again, when $p=1$ and as $p \rightarrow \infty$ we recover the squares $D$ and $E$. Figure 4 shows several of these sets, with $B_{1 / 2}$ the innermost and $B_{\infty}$ the outermost. (We remark that the boundary of $B_{1 / 2}$ is also closely related to Vershik's curve $C_{1}$.) In some cases, we can explicitly compute the limiting curves of the $\tilde{P}_{Q}\left(B_{p}\right)$, and in these cases the limiting curves are again piecewise algebraic. In all cases, we obtain a parametric representation of the limiting curves, and we suspect that they are not in general piecewise algebraic. This family of examples is investigated in Section 5. 
We note in passing that one can consider the problem of generalizing Vershik's theorem to domains other than the unit square $E$. Given a set $S \in \mathbb{R}^{2}$, choose a polygon $P$ at random uniformly from among all convex polygons whose vertices lie in the set $\left(\frac{1}{Q} \mathbb{Z}\right)^{2} \cap S$. Is there a curve $V(S)$ such that, with probability approaching 1 as $Q$ tends to infinity, the polygon $P$ lies within any prescribed open neighborhood of $V(S)$ ? Vershik's result is that $V(E)=C_{1}$; it seems likely that $V(D)$ is the curve $C$ scaled by a factor of $\frac{3}{4}$, so that it passes through the points $\left( \pm \frac{1}{2}, \pm \frac{1}{2}\right)$. In general, it would be interesting to search for a connection between these generalized "Vershik curves" $V(S)$ and the limiting curves of familes of generalized Jarník polygons $\left\{P_{Q}\left(S^{\prime}\right)\right\}$ for appropriate sets $S$ and $S^{\prime}$.

\section{ThE ORIGINAL JARNÍK POLYGONS}

Because of the eight-fold symmetry of the Jarník polygons, we need only consider the portion of $P_{Q}$ starting from the edge corresponding to the vector $(1,0)$ and ending with the edge corresponding to the vector $(1,1)$; we call this eighth-portion the fundamental arc of $P_{Q}$, as the entire polygon $P_{Q}$ is generated from the fundamental arc under the action of the dihedral group of order eight. For example, the fundamental arc of $P_{4}$ consists of the seven edges defined by the first eight vertices in the list (2). Given any curve $C$ with eight-fold dihedral symmetry about the origin (including the scaled Jarník polygons $\tilde{P}_{Q}$ and their generalizations), we shall also refer to the eighth-portion of the curve lying in the wedge $\{(x, y): x>0, y<-x\}$ as the fundamental arc of $C$.

For any real number $\lambda \in[0,1]$ we define $V_{Q}(\lambda)$ to be the set of all vectors in $V_{Q}$ with positive coordinates and slope not exceeding $\lambda$. The sum of all the vectors in $V_{Q}(\lambda)$ corresponds to a particular vertex on the fundamental arc of $P_{Q}$. If we translate $P_{Q}$ so that the right-hand endpoint of the edge corresponding to the vector $(1,0)$ is at the origin, as in (2), then the coordinates $(X(Q, \lambda), Y(Q, \lambda))$ of this vertex are given by the formulas

$$
X(Q, \lambda)=\sum_{q \leq Q} q \sum_{\substack{a \leq \lambda q \\ \operatorname{gcd}(q, a)=1}} 1 \text { and } Y(Q, \lambda)=\sum_{q \leq Q} \sum_{\substack{a \leq \lambda q \\ \operatorname{gcd}(q, a)=1}} a
$$

For example, we see from equation (11) that $V_{4}\left(\frac{1}{\sqrt{3}}\right)$ consists of the three vectors $(4,1),(3,1)$, and $(2,1)$, and hence $\left(X\left(4, \frac{1}{\sqrt{3}}\right), Y\left(4, \frac{1}{\sqrt{3}}\right)\right)$ is the vertex $(9,3)$ of $P_{4}$.

The following asymptotic evaluation of $(X(Q, \lambda)$ and $Y(Q, \lambda))$ is the key to our calculation.

Lemma 3. We have

$$
X(Q, \lambda)=\frac{2 \lambda Q^{3}}{\pi^{2}}+O\left(Q^{2} \log Q\right) \quad \text { and } \quad Y(Q, \lambda)=\frac{\lambda^{2} Q^{3}}{\pi^{2}}+O\left(Q^{2} \log Q\right)
$$

uniformly for $Q \geq 2$ and $0 \leq \lambda \leq 1$.

Proof. Recall the definition of the Möbius mu-function

$$
\mu(n)= \begin{cases}1, & \text { if } n=1, \\ (-1)^{r}, & \text { if } n=p_{1} p_{2} \ldots p_{r}, \text { where the } p_{i} \text { are distinct primes, } \\ 0, & \text { if the square of any prime divides } n\end{cases}
$$


The well-known Möbius inversion formula is based on the characteristic property of $\mu$ :

$$
\sum_{d \mid n} \mu(d)= \begin{cases}1, & \text { if } n=1 \\ 0, & \text { if } n>1\end{cases}
$$

It is also well known that $\sum_{q \geq 1} \mu(q) / q^{2}=1 / \zeta(2)=6 / \pi^{2}$, where $\zeta$ denotes the Riemann zeta-function. We shall use the truncated version of this identity:

$$
\sum_{q \leq Q} \frac{\mu(q)}{q^{2}}=\frac{6}{\pi^{2}}+O\left(\frac{1}{Q}\right)
$$

which follows easily by a trivial estimation of the tail by $\sum_{q>Q} 1 / q^{2}$.

Property (3) allows us to write

$$
X(Q, \lambda)=\sum_{q \leq Q} q \sum_{a \leq \lambda q}\left(\sum_{d \mid \operatorname{gcd}(q, a)} \mu(d)\right)=\sum_{d \leq Q} \mu(d) \sum_{\substack{q \leq Q \\ d \mid q}} q \sum_{\substack{a \leq \lambda q \\ d \mid a}} 1 .
$$

Writing $a=b d$ and $q=r d$, we have

$$
\begin{aligned}
X(Q, \lambda) & =\sum_{d \leq Q} \mu(d) \sum_{r \leq Q / d} d r \sum_{b \leq \lambda r} 1 \\
& =\sum_{d \leq Q} d \mu(d) \sum_{r \leq Q / d} r(\lambda r+O(1)) \\
& =\lambda \sum_{d \leq Q} d \mu(d) \sum_{r \leq Q / d} r^{2}+O\left(\sum_{d \leq Q} d \sum_{r \leq Q / d} r\right) \\
& =\lambda \sum_{d \leq Q} d \mu(d)\left(\frac{1}{3}\left(\frac{Q}{d}\right)^{3}+O\left(\left(\frac{Q}{d}\right)^{2}\right)\right)+O\left(\sum_{d \leq Q} d\left(\frac{Q}{d}\right)^{2}\right) \\
& =\frac{\lambda Q^{3}}{3} \sum_{d \leq Q} \frac{\mu(d)}{d^{2}}+O\left(Q^{2} \sum_{d \leq Q} \frac{1}{d}\right) .
\end{aligned}
$$

Equation (4) now allows us to conclude that

$$
X(Q, \lambda)=\frac{\lambda Q^{3}}{3}\left(\frac{6}{\pi^{2}}+O\left(\frac{1}{Q}\right)\right)+O\left(Q^{2} \log Q\right)=\frac{2 \lambda Q^{3}}{\pi^{2}}+O\left(Q^{2} \log Q\right),
$$

as claimed. 
In the same way we see that

$$
\begin{aligned}
Y(Q, \lambda) & =\sum_{q \leq Q} \sum_{a \leq \lambda q} a\left(\sum_{d \mid \operatorname{gcd}(q, a)} \mu(d)\right) \\
& =\sum_{d \leq Q} \mu(d) \sum_{\substack { q \leq Q \\
\begin{subarray}{c}{|| q \\
a \leq \lambda q{ q \leq Q \\
\begin{subarray} { c } { | | q \\
a \leq \lambda q } }\end{subarray}} a \\
& =\sum_{d \leq Q} \mu(d) \sum_{r \leq Q / d} \sum_{b \leq \lambda r} d b \\
& =\sum_{d \leq Q} d \mu(d) \sum_{r \leq Q / d}\left(\frac{1}{2}(\lambda r)^{2}+O(\lambda r)\right) \\
& =\frac{\lambda^{2}}{2} \sum_{d \leq Q} d \mu(d) \sum_{r \leq Q / d} r^{2}+O\left(\sum_{d \leq Q} d \sum_{r \leq Q / d} r\right) .
\end{aligned}
$$

At this point, a direct comparison to the middle line of equation (5) yields

$$
Y(Q, \lambda)=\frac{\lambda^{2} Q^{3}}{\pi^{2}}+O\left(Q^{2} \log Q\right)
$$

as claimed.

We can now prove Theorem 1. Define $R(Q)=X(Q, 1)+Y(Q, 1)-1 / 2$, so that $R(Q)=3 Q^{3} / \pi^{2}+O\left(Q^{2} \log Q\right)$ by Lemma 3. If we translate $P_{Q}$ so that the midpoint of the edge corresponding to the vector $(1,0)$ is at the point $(0,-R(Q))$, then the center of $P_{Q}$ will be at the origin due to the symmetries of $P_{Q}$; we then obtain $\tilde{P}_{Q}$ by scaling by the factor $1 / R(Q)$. If $(\tilde{X}(Q, \lambda), \tilde{Y}(Q, \lambda))$ is the vertex of $\tilde{P}_{Q}$ corresponding to the vertex $(X(Q, \lambda), Y(Q, \lambda))$ of $P_{Q}$, then

$$
\begin{aligned}
& \tilde{X}(Q, \lambda)=\frac{X(Q, \lambda)+1 / 2}{R(Q)}=\frac{2 \lambda}{3}+O\left(\frac{\log Q}{Q}\right), \\
& \tilde{Y}(Q, \lambda)=\frac{Y(Q, \lambda)-R(Q)}{R(Q)}=\frac{\lambda^{2}}{3}-1+O\left(\frac{\log Q}{Q}\right),
\end{aligned}
$$

using Lemma 3 In particular, when $Q$ is large enough, the vertices on the fundamental arc of $\tilde{P}_{Q}$ lie within $\varepsilon / 2$ (say) of the arc parametrized by $\left(2 \lambda / 3, \lambda^{2} / 3-1\right)$ with $0 \leq \lambda \leq 1$. This parametric curve is precisely the arc of the parabola $y=3 x^{2} / 4-1$ from $x=0$ to $x=2 / 3$, which is an eighth-portion of the curve $C$. Moreover, the lengths of the edges of $\tilde{P}_{Q}$ are $O\left(1 / Q^{2}\right)$, and so every point on the fundamental arc of $\tilde{P}_{Q}$ lies within $\varepsilon$ of $C$ when $Q$ is large enough. Finally, because of the symmetries of $C$ and the $\tilde{P}_{Q}$, we see that the entire polygon $\tilde{P}_{Q}$ lies within an $\varepsilon$-neighborhood of $C$ when $Q$ is large enough. This establishes Theorem 1.

\section{Polygons Defined By the unit diamond}

We begin by looking at the derivation of Lemma 3 from another viewpoint. For any real number $0 \leq \lambda \leq 1$, define $E(\lambda)=\{(x, y) \in E: x>0,0<y \leq \lambda x\}$. The inner double sum in the first line of equation (5) is written as a sum over lattice points in a large wedge, but we may reinterpret it as a sum over $\left(\frac{1}{Q} \mathbb{Z}\right)^{2} \cap E(\lambda)$ by 
writing

$$
\sum_{d \leq Q} \mu(d) \sum_{r \leq Q / d} d r \sum_{b \leq \lambda r} 1=Q^{3} \sum_{d \leq Q} \frac{\mu(d)}{d^{2}}\left(\left(\frac{d}{Q}\right)^{2} \sum_{r: 0<d r / Q \leq 1} \frac{d r}{Q} \sum_{b: 0<d b / Q \leq \lambda d r / Q} 1\right) .
$$

Notice that the quantity in parentheses is a Riemann sum approximating the integral

$$
\int_{0}^{1} x \int_{0}^{\lambda x} d y d x=\iint_{E(\lambda)} x d x d y
$$

and in fact (since the integrand $x$ has bounded first derivatives) the error in making this approximation will be proportional to the mesh size, which is $O(d / Q)$. Therefore

$$
\begin{aligned}
X(Q, \lambda) & =Q^{3} \sum_{d \leq Q} \frac{\mu(d)}{d^{2}}\left(\iint_{E(\lambda)} x d x d y+O\left(\frac{d}{Q}\right)\right) \\
& =Q^{3}\left(\iint_{E(\lambda)} x d x d y\right) \sum_{d \leq Q} \frac{\mu(d)}{d^{2}}+O\left(Q^{2} \sum_{d \leq Q} \frac{|\mu(d)|}{d}\right) \\
& =\frac{Q^{3}}{\zeta(2)} \iint_{E(\lambda)} x d x d y+O\left(Q^{2} \log Q\right) .
\end{aligned}
$$

This is in agreement with Lemma 3, as $\zeta(2)=\pi^{2} / 6$ and

$$
\iint_{E(\lambda)} x d x d y=\int_{0}^{\lambda}\left(\int_{y / \lambda}^{1} x d x\right) d y=\frac{\lambda}{3} .
$$

Similar remarks apply to $Y(Q, \lambda)$.

We shall use a similar approach for the generalized Jarník polygons $P_{Q}(S)$. For any real number $\lambda \in[0,1]$ we define $V_{Q}(S, \lambda)$ to be the set of all vectors in $V_{Q}(S)$ with positive coordinates and slope not exceeding $\lambda$. The sum of all the vectors in $V_{Q}(S, \lambda)$ corresponds to a particular vertex on the fundamental arc of $P_{Q}(S)$. If we translate $P_{Q}(S)$ so that the right-hand endpoint of the edge corresponding to the vector $(1,0)$ is at the origin, then the coordinates $\left(X_{S}(Q, \lambda), Y_{S}(Q, \lambda)\right)$ of this vertex are given by the formulas

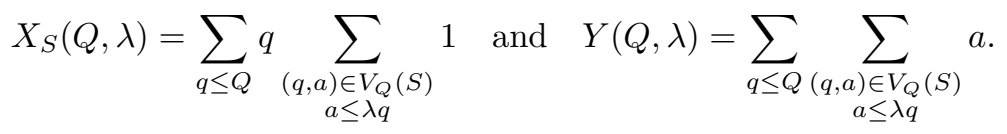

If we define $S(\lambda)=\{(x, y) \in S: x>0,0<y \leq \lambda x\}$, then the same argument as above allows us to conclude that

$$
X_{S}(Q, \lambda) \sim \frac{Q^{3}}{\zeta(2)} \iint_{S(\lambda)} x d x d y \quad \text { and } \quad Y_{S}(Q, \lambda) \sim \frac{Q^{3}}{\zeta(2)} \iint_{S(\lambda)} y d x d y .
$$

This depends of course on $S$ being a "reasonable" set. For the sets $S$ that we shall consider, the asymptotic formulas (6) do in fact hold, with error terms that are $O\left(Q^{2} \log Q\right)$.

We also use the definitions $R_{S}(Q)=X_{S}(Q, 1)+Y_{S}(Q, 1)-1 / 2$ and

$$
\tilde{X}_{S}(Q, \lambda)=\frac{X_{S}(Q, \lambda)+1 / 2}{R_{S}(Q)}, \quad \tilde{Y}_{S}(Q, \lambda)=\frac{Y_{S}(Q, \lambda)-R_{S}(Q)}{R_{S}(Q)},
$$

so that $\left(\tilde{X}_{S}(Q, \lambda), \tilde{Y}_{S}(Q, \lambda)\right)$ will be the coordinates of the corresponding vertex on the fundamental arc of the scaled generalized Jarník polygon $\tilde{P}_{Q}(S)$, the translation 
and scaling chosen so that the center of $\tilde{P}_{Q}(S)$ is the origin and the points $( \pm 1,0)$ and $(0, \pm 1)$ are midpoints of edges of $\tilde{P}_{Q}(S)$.

We now implement this approach with $S$ equaling the unit diamond $D$ to prove Theorem 2. Again, due to the symmetries of $C_{1}$ and the $P_{Q}(D)$, it suffices to show that the fundamental $\operatorname{arcs}$ of the $P_{Q}(D)$ tend to the fundamental arc of $C_{1}$. Given $0 \leq \lambda \leq 1$, the set $D(\lambda)$ is the same as $\{(x, y): x>0, x+y \leq 1, y \leq \lambda x\}=$ $\{(x, y): 0<y \leq \lambda /(1+\lambda), y / \lambda \leq x \leq 1-y\}$. Therefore

$$
\begin{aligned}
& \iint_{D(\lambda)} x d x d y=\int_{0}^{\lambda /(1+\lambda)}\left(\int_{y / \lambda}^{1-y} x d x\right) d y=\frac{\lambda(2+\lambda)}{6(1+\lambda)^{2}} \\
& \iint_{D(\lambda)} y d x d y=\int_{0}^{\lambda /(1+\lambda)} y\left(\int_{y / \lambda}^{1-y} d x\right) d y=\frac{\lambda^{2}}{6(1+\lambda)^{2}} .
\end{aligned}
$$

Using these evaluations in equation (6), we see that

$$
X_{D}(Q, \lambda) \sim \frac{Q^{3} \lambda(2+\lambda)}{\pi^{2}(1+\lambda)^{2}}, \quad Y_{D}(Q, \lambda) \sim \frac{Q^{3} \lambda^{2}}{\pi^{2}(1+\lambda)^{2}}
$$

as $\zeta(2)=\pi^{2} / 6$. This implies that $R_{D}(Q) \sim Q^{3} / \pi^{2}$, and so

$$
\begin{aligned}
& \tilde{X}_{D}(Q, \lambda)=\frac{X_{D}(Q, \lambda)+1 / 2}{R_{D}(Q)} \sim \frac{\lambda(2+\lambda)}{(1+\lambda)^{2}}, \\
& \tilde{Y}_{D}(Q, \lambda)=\frac{Y_{D}(Q, \lambda)-R_{D}(Q)}{R_{D}(Q)} \sim \frac{-(2 \lambda+1)}{(1+\lambda)^{2}} .
\end{aligned}
$$

If we set $x=\frac{\lambda(2+\lambda)}{(1+\lambda)^{2}}$ and $y=\frac{-(2 \lambda+1)}{(1+\lambda)^{2}}$, it is easy to check that $\sqrt{1-|x|}+\sqrt{1-|y|}=$ 1 , and hence the curve parametrized by $\left(\frac{\lambda(2+\lambda)}{(1+\lambda)^{2}}, \frac{-(2 \lambda+1)}{(1+\lambda)^{2}}\right)$ with $0 \leq \lambda \leq 1$ is precisely the fundamental arc of $C_{1}$. This establishes Theorem 2 .

\section{Polygons defined by octagons}

Recall that for any positive real number $\delta$, we defined $O_{\delta}$ to be the octagon with vertices at $( \pm 1,0)$ and $(0, \pm 1)$ and the four points $\left( \pm \frac{\delta}{1+\delta}, \pm \frac{\delta}{1+\delta}\right)$. We can use the same strategy to calculate the limiting curve of the generalized Jarník polygons generated from the sets $O_{\delta}$. Define $C_{\delta}$ to be the curve with eight-fold dihedral symmetry whose fundamental arc is

$$
\left\{(x, y): 0 \leq x \leq \frac{2 \delta+1}{3 \delta+1}, 4 \delta(1+\delta)^{2}(y+1)=(1+3 \delta)(\delta x+y+1)^{2}\right\} .
$$

This arc is part of a parabola whose axis of symmetry has slope $-\delta$ and whose vertex is $\left(\frac{(1+\delta)^{2}\left(1+2 \delta^{2}\right)}{(1+3 \delta)\left(1+\delta^{2}\right)^{2}}, \frac{-\left(1+2 \delta+5 \delta^{3}+\delta^{4}+3 \delta^{5}\right)}{(1+3 \delta)\left(1+\delta^{2}\right)^{2}}\right)$, as it turns out. The endpoints of this parabolic arc are $(0,-1)$ and $\left(\frac{2 \delta+1}{3 \delta+1},-\frac{2 \delta+1}{3 \delta+1}\right)$. In particular, each $C_{\delta}$ is piecewise algebraic, and one can check that each $C_{\delta}$ is twice differentiable at the eight symmetry points $( \pm 1,0),(0, \pm 1)$, and $\left( \pm \frac{2 \delta+1}{3 \delta+1}, \pm \frac{2 \delta+1}{3 \delta+1}\right)$. Figure 5 shows several of these curves, with $C_{1 / 4}$ the outermost and $C_{\infty}$ the innerermost; the points $\left( \pm \frac{2}{3}, \pm \frac{2}{3}\right)$ and $\left( \pm \frac{3}{4}, \pm \frac{3}{4}\right)$, which lie on $C_{\infty}$ and $C_{1}$, respectively, are also indicated.

Theorem 4. For every positive real number $\delta$, the scaled generalized Jarnik polygons $\left\{\tilde{P}_{Q}\left(O_{\delta}\right)\right\}$ converge to $C_{\delta}$.

Note that when $\delta=1$, the octagon $O_{1}$ degenerates to the unit diamond $D$; in this case, the equation of the parabola in equation (8) is equivalent to $4(y+1)=$ $(x+y+1)^{2}$, which turns out to be another way to define the fundamental arc of 

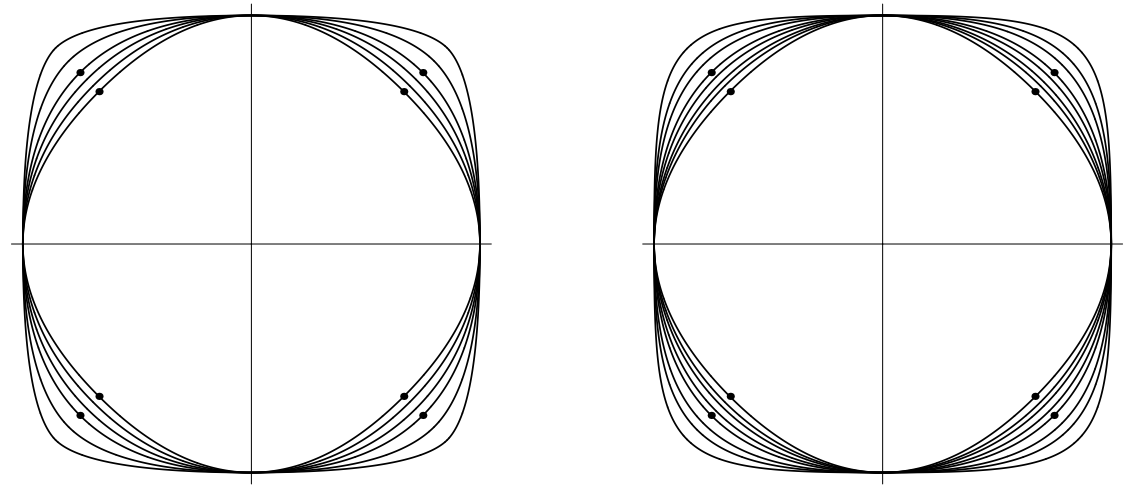

Figure 5. The limiting curves $C_{\delta}$, left, and $C_{p}^{\prime}$, right

the curve $C_{1}$. Therefore the notation $C_{\delta}$ is consistent with our earlier definition of $C_{1}$, and Theorem 4 is consistent with Theorem 2. Also, as $\delta$ tends to infinity, the octagons $O_{\delta}$ converge to the unit square $E$, while the equation of the parabola in equation (8) tends to $4(y+1)=3 x^{2}$, which is the equation defining the fundamental arc of $C$. Therefore Theorem 4 has Theorem 1 as a limiting case as well. It can also be checked that $C_{\delta}$ tends towards the boundary of the unit square $E$ as $\delta$ decreases to zero.

We prove Theorem 4 using the same approach as the proof of Theorem 2 in the previous section, showing the important steps while supressing the algebraic details of the computations. Given $0 \leq \lambda \leq 1$, we have by equation (6)

$$
\begin{aligned}
X_{O_{\delta}}(Q, \lambda) & \sim \frac{Q^{3}}{\zeta(2)} \iint_{O_{\delta}(\lambda)} x d x d y \\
& \sim \frac{Q^{3}}{\zeta(2)} \int_{0}^{\delta \lambda /(\delta+\lambda)}\left(\int_{y / \lambda}^{1-y / \delta} x d x\right) d y=\frac{Q^{3}}{\pi^{2}} \frac{\delta \lambda(2 \delta+\lambda)}{(\delta+\lambda)^{2}}, \\
Y_{O_{\delta}}(Q, \lambda) & \sim \frac{Q^{3}}{\zeta(2)} \iint_{O_{\delta}(\lambda)} y d x d y \\
& \sim \frac{Q^{3}}{\zeta(2)} \int_{0}^{\delta \lambda /(\delta+\lambda)} y\left(\int_{y / \lambda}^{1-y / \delta} d x\right) d y=\frac{Q^{3}}{\pi^{2}} \frac{\delta^{2} \lambda^{2}}{(\delta+\lambda)^{2}} .
\end{aligned}
$$

This implies that $R_{O_{\delta}}(Q) \sim \frac{Q^{3} \delta(3 \delta+1)}{\pi^{2}(\delta+1)^{2}}$, and so

$$
\begin{aligned}
& \tilde{X}_{O_{\delta}}(Q, \lambda)=\frac{X_{O_{\delta}}(Q, \lambda)+1 / 2}{R_{O_{\delta}}(Q)} \sim \frac{\lambda(2 \delta+\lambda)(\delta+1)^{2}}{(\delta+\lambda)^{2}(3 \delta+1)}, \\
& \tilde{Y}_{O_{\delta}}(Q, \lambda)=\frac{Y_{O_{\delta}}(Q, \lambda)-R_{O_{\delta}}(Q)}{R_{O_{\delta}}(Q)} \sim \frac{\delta \lambda^{2}(\delta+1)^{2}}{(\delta+\lambda)^{2}(3 \delta+1)}-1 .
\end{aligned}
$$

If we set

$$
x=\frac{\lambda(2 \delta+\lambda)(\delta+1)^{2}}{(\delta+\lambda)^{2}(3 \delta+1)} \quad \text { and } \quad y=\frac{\delta \lambda^{2}(\delta+1)^{2}}{(\delta+\lambda)^{2}(3 \delta+1)}-1,
$$

we can check that $(x, y)$ satisfies the polynomial relation (8), and hence the curve parametrized by $\left(\frac{\lambda(2 \delta+\lambda)(\delta+1)^{2}}{(\delta+\lambda)^{2}(3 \delta+1)}, \frac{\delta \lambda^{2}(\delta+1)^{2}}{(\delta+\lambda)^{2}(3 \delta+1)}-1\right)$ with $0 \leq \lambda \leq 1$ is precisely the fundamental arc of $C_{\delta}$. This establishes Theorem 4 . 


\section{Polygons Defined By Unit BALls}

Recall that for any positive real number $p$, we defined $B_{p}$ to be the set $\left\{|x|^{p}+\right.$ $\left.|y|^{p} \leq 1\right\}$, which we refer to as the "unit $\ell^{p}$-ball" (an abuse of terminology when $p<1)$. We also need the standard notation $B(a, b)$ for the Euler beta function

$$
B(a, b)=\int_{0}^{1} t^{a-1}(1-t)^{b-1} d t=\Gamma(a) \Gamma(b) / \Gamma(a+b)
$$

as well as its relatives, the incomplete beta function

$$
B_{z}(a, b)=\int_{0}^{z} t^{a-1}(1-t)^{b-1} d t
$$

and the regularized incomplete beta function

$$
I_{z}(a, b)=B_{z}(a, b) / B(a, b) .
$$

Let $p$ be a positive real number, which we regard as fixed. When $0 \leq \lambda \leq 1$, set $\mu=\mu(\lambda)=\frac{\lambda^{p}}{1+\lambda^{p}}$, and define $C_{p}^{\prime}$ to be the curve with eight-fold dihedral symmetry whose fundamental arc is given parametrically by

$$
\left(I_{\mu}\left(\frac{1}{p}, 1+\frac{2}{p}\right)-\frac{p \lambda\left(1+\lambda^{p}\right)^{-3 / p}}{2 B\left(\frac{1}{p}, \frac{2}{p}\right)}, I_{\mu}\left(\frac{2}{p}, 1+\frac{1}{p}\right)-\frac{p \lambda^{2}\left(1+\lambda^{p}\right)^{-3 / p}}{B\left(\frac{1}{p}, \frac{2}{p}\right)}-1\right), \quad 0 \leq \lambda \leq 1 .
$$

Figure 5 shows several of these curves, with $C_{1 / 3}^{\prime}$ the outermost and $C_{\infty}^{\prime}$ the innerermost; the points $\left( \pm \frac{2}{3}, \pm \frac{2}{3}\right)$ and $\left( \pm \frac{3}{4}, \pm \frac{3}{4}\right)$, which lie on $C_{\infty}^{\prime}$ and $C_{1}^{\prime}$, respectively, are also indicated.

Theorem 5. For every positive real number $p$, the scaled generalized Jarnik polygons $\left\{\tilde{P}_{Q}\left(B_{p}\right)\right\}$ converge to $C_{p}^{\prime}$.

Although the curves $C_{p}^{\prime}$ are in general rather inscrutable, it can be shown that they are all twice differentiable at the eight points of symmetry and infinitely differentiable everywhere else. In the special case $p=1$, the ball $B_{1}$ is exactly the unit diamond $D$. The parametric representation (9) of the fundamental arc of $C_{1}^{\prime}$ reduces to $\left(\frac{\lambda(2+\lambda)}{(1+\lambda)^{2}}, \frac{-(2 \lambda+1)}{(1+\lambda)^{2}}\right)$, which by equation (17) is the parametric representation of the fundamental arc of $C_{1}$. (Again in this section, we suppress the details of many of our calculations.) Therefore Theorem 5 is consistent with Theorem 2.

It can also be shown that as $p$ tends to infinity, the parametric representation (9) of $C_{p}^{\prime}$ approaches $\left(2 \lambda / 3, \lambda^{2} / 3-1\right)$, which is the parametrization of the fundamental arc of $C$. Therefore Theorem 5 has Theorem 1 as a limiting case as well. Again, it can be checked that $C_{p}^{\prime}$ tends towards the boundary of the unit square $E$ as $p$ decreases to zero.

The case $p=2$ is also special, since the domain $B_{2}$ (the unit disk) has complete rotational symmetry. Indeed, the parametric representation (9) of the fundamental arc of $C_{2}^{\prime}$ reduces to $\left(\frac{\lambda}{\sqrt{1+\lambda^{2}}},-\frac{1}{\sqrt{1+\lambda^{2}}}\right)$, which is the fundamental arc of the unit circle. Thus the limiting curve of the generalized Jarník polygons formed from the vectors in $V_{Q}\left(B_{2}\right)$ is simply the unit circle, not surprisingly.

When $p$ is the reciprocal of a positive integer, the regularized incomplete beta functions are simply indefinite integrals of polynomials. Therefore in these cases, the parametric representation (9) of the fundamental arc of $C_{p}^{\prime}$ can be written as rational functions of $\lambda^{p}$. In particular, these particular curves $C_{p}^{\prime}$ are piecewise algebraic, and in principal one can calculate the algebraic equation defining the 
fundamental arc. For example, when $p=1 / 2$ the parametric representation (9) of the fundamental arc of $C_{1 / 2}^{\prime}$ reduces to

$$
\left(\frac{\lambda\left(10+10 \lambda^{1 / 2}+5 \lambda+\lambda^{3 / 2}\right)}{\left(1+\lambda^{1 / 2}\right)^{5}}, \frac{-\left(1+5 \lambda^{1 / 2}+10 \lambda+10 \lambda^{3 / 2}\right)}{\left(1+\lambda^{1 / 2}\right)^{5}}\right), \quad 0 \leq \lambda \leq 1,
$$

and the coordinates $(x, y)$ of this parametrization satisfy the irreducible polynomial relation

$$
\begin{gathered}
-45253+86140 x-37030 x^{2}-3220 x^{3}-765 x^{4}+128 x^{5}-86140 y+169060 x y \\
-80340 x^{2} y-1940 x^{3} y-640 x^{4} y-37030 y^{2}+80340 x y^{2}-44590 x^{2} y^{2}+1280 x^{3} y^{2} \\
+3220 y^{3}-1940 x y^{3}-1280 x^{2} y^{3}-765 y^{4}+640 x y^{4}-128 y^{5}=0 .
\end{gathered}
$$

We establish Theorem 5 using our now familiar technique. For any $0 \leq \lambda \leq 1$, equation (6) gives

$$
\begin{aligned}
X_{B_{p}}(Q, \lambda) & \sim \frac{Q^{3}}{\zeta(2)} \iint_{B_{p}(\lambda)} x d x d y \\
& =\frac{Q^{3}}{\zeta(2)} \int_{0}^{\mu^{1 / p}}\left(\int_{y / \lambda}^{\left(1-y^{p}\right)^{1 / p}} x d x\right) d y \\
& =\frac{Q^{3}}{\zeta(2)}\left(\frac{1}{2 p} B_{\mu}\left(\frac{1}{p}, 1+\frac{2}{p}\right)-\frac{1}{6} \lambda\left(1+\lambda^{p}\right)^{-3 / p}\right), \\
Y_{B_{p}}(Q, \lambda) & \sim \frac{Q^{3}}{\zeta(2)} \iint_{B_{p}(\lambda)} y d x d y \\
& =\frac{Q^{3}}{\zeta(2)} \int_{0}^{\mu^{1 / p}} y\left(\int_{y / \lambda}^{\left(1-y^{p}\right)^{1 / p}} d x\right) d y \\
& =\frac{Q^{3}}{\zeta(2)}\left(\frac{1}{p} B_{\mu}\left(\frac{2}{p}, 1+\frac{1}{p}\right)-\frac{1}{3} \lambda^{2}\left(1+\lambda^{p}\right)^{-3 / p}\right) .
\end{aligned}
$$

This implies that

$$
\begin{aligned}
R_{B_{p}}(Q) \sim \frac{Q^{3}}{\zeta(2)} \frac{1}{3 p} B\left(\frac{1}{p}, \frac{2}{p}\right) & =\frac{Q^{3}}{\zeta(2)} \frac{1}{2 p} B\left(\frac{1}{p}, 1+\frac{2}{p}\right) \\
& =\frac{Q^{3}}{\zeta(2)} \frac{1}{p} B\left(\frac{2}{p}, 1+\frac{1}{p}\right),
\end{aligned}
$$

and so after much calculation we see that

$$
\begin{aligned}
& \tilde{X}_{B_{p}}(Q, \lambda) \sim I_{\mu}\left(\frac{1}{p}, 1+\frac{2}{p}\right)-\frac{p \lambda}{2\left(1+\lambda^{p}\right)^{3 / p} B\left(\frac{1}{p}, \frac{2}{p}\right)}, \\
& \tilde{Y}_{B_{p}}(Q, \lambda) \sim-\left(I_{1-\mu}\left(\frac{1}{p}, 1+\frac{2}{p}\right)-\frac{p \lambda^{2}}{2\left(1+\lambda^{p}\right)^{3 / p} B\left(\frac{1}{p}, \frac{2}{p}\right)}\right),
\end{aligned}
$$

which is exactly the parametric definition (9) of the fundamental arc of $C_{p}^{\prime}$. This establishes Theorem [5]

\section{Local CURVATURES}

Given a vertex $v$ of any polygon $P$, we define the radius of curvature of $P$ at $v$ to be the radius of the circle passing through $v$ and its two neighbors. We quantify the local curvatures of the Jarník polygons (as originally defined) in the following way. 

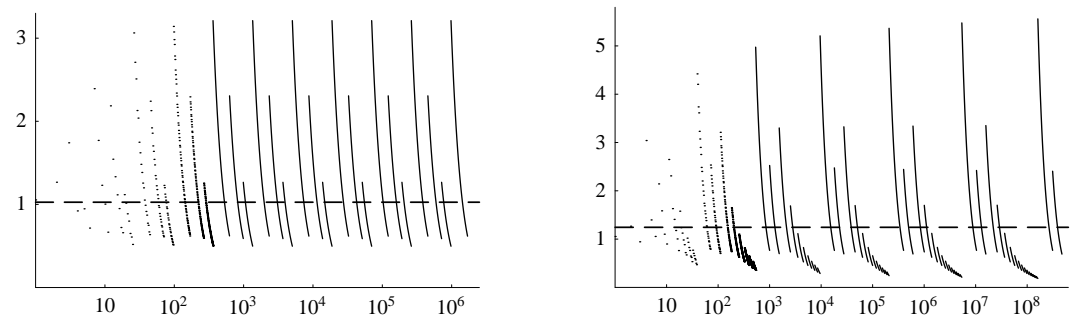

Figure 6 . The local radii of curvature $\tilde{r}_{Q}(\lambda)$ for $\lambda=\frac{1}{\sqrt{3}}$, left, and $\lambda=e-2$, right

To each irrational number $0<\lambda<1$, we associate the unique vertex $v_{Q}(\lambda)$ on the fundamental arc of $P_{Q}$ such that $\lambda$ lies between the slopes of the two edges adjacent to $v_{Q}(\lambda)$. We then define $r_{Q}(\lambda)$ to be the radius of curvature of $P_{Q}$ at $v(Q, \lambda)$. This description is not well-defined for rational numbers $\lambda$, but we can speak of $r_{Q}\left(\lambda^{+}\right)$ and $r_{Q}\left(\lambda^{-}\right)$. For example, from the list (2) we see that $r_{Q}\left(\frac{1}{\sqrt{3}}\right)$ is the radius of the circle passing through the points $(7,2),(9,3)$, and $(12,5)$, which turns out to be $\sqrt{1105 / 2}$. We also have $r_{Q}\left(\frac{1}{2}^{+}\right)=\sqrt{1105 / 2}$, but $r_{Q}\left(\frac{1}{2}^{-}\right)=5 \sqrt{29 / 2}$.

After scaling the Jarník polygons, the radius of curvature $\tilde{r}_{Q}(\lambda)$ at the corresponding vertex of $\tilde{P}_{Q}$ is simply $r_{Q}(\lambda) / R(Q)$. It would be tidy if, as $Q$ grew large, the local radii of curvature $\tilde{r}_{Q}(\lambda)$ would converge to the radius of curvature of the limiting curve $C$ at the corresponding point $\left(2 \lambda / 3, \lambda^{2} / 3-1\right)$, which turns out to be $\frac{2}{3}\left(1+\lambda^{2}\right)^{3 / 2}$. However, not only does $\left\{\tilde{r}_{Q}(\lambda)\right\}$ never converge to $\frac{2}{3}\left(1+\lambda^{2}\right)^{3 / 2}$, but in fact $\left\{\tilde{r}_{Q}(\lambda)\right\}$ fails to converge at all for most $\lambda$, and the manner in which it fails to converge depends upon the diophantine approximation properties of $\lambda$. In Figure 6 we have plotted these local radii of curvature $\tilde{r}_{Q}(\lambda)$ as functions of $Q$ (represented on the horizontal axis in logarithmic scale) for two interesting examples of irrational numbers, $\lambda=\frac{1}{\sqrt{3}}$ and $\lambda=e-2$, with the horizontal dashed line indicating the value $\frac{2}{3}\left(1+\lambda^{2}\right)^{3 / 2}$ in each case.

We recall some notation and standard facts from the theory of diophantine approximation and continued fractions. The Farey fractions of order $Q$ are defined to be the rational numbers in $[0,1]$ with denominator not exceeding $Q$, listed in increasing order. For example, the Farey fractions of order 4 are $\left\{\frac{0}{1}, \frac{1}{4}, \frac{1}{3}, \frac{1}{2}, \frac{2}{3}, \frac{3}{4}, \frac{1}{1}\right\}$. There is a one-to-one correspondence between the Farey fractions $\frac{a}{q}$ of order $Q$ and the vectors $(q, a)$ in the "fundamental arc" of $V_{Q}$, as we see from the list (1) when $Q=4$. If $\frac{a_{1}}{q_{1}}$ and $\frac{a_{2}}{q_{2}}$ are consecutive Farey fractions, it is known (see [4, Section 6.1]) that $a_{2} q_{1}-a_{1} q_{2}=1$.

Let $\lambda$ be an irrational number in $(0,1)$ with continued fraction expansion $\left[0 ; b_{1}, b_{2}, \ldots\right]$, where the $b_{n}$ are positive integers. Define sequences $\left\{h_{n}\right\},\left\{k_{n}\right\}$ of positive integers by

$$
\begin{aligned}
h_{0}=1, & h_{1}=0, \quad h_{n+1}=b_{n} h_{n}+h_{n-1} \quad(n \geq 1), \\
k_{0}=0, & k_{1}=1, \quad k_{n+1}=b_{n} k_{n}+k_{n-1} \quad(n \geq 1) .
\end{aligned}
$$

The $\left\{h_{n} / k_{n}\right\}$ are the convergents to $\lambda$. It is immediate that for any real number $Q \geq k_{2}=b_{1}$, there are a unique index $n \geq 2$ and a unique integer $1 \leq j \leq b_{n}$ such that $j k_{n}+k_{n-1} \leq Q<(j+1) k_{n}+k_{n-1}$. It is known that in the set of Farey 
fractions of order $Q$, the number $\lambda$ lies between two fractions whose denominators are $k_{n}$ and $j k_{n}+k_{n-1}$ in this notation. (See [4, Section 7.5, Problem 5]. Fractions of the form $\left(j h_{n}+h_{n-1}\right) /\left(j k_{n}+k_{n-1}\right)$ with $1 \leq j<b_{n}$ are called the secondary convergents to $\lambda$.)

For example, if $\lambda=\frac{1}{\sqrt{3}}=[0 ; 1,1,2,1,2,1,2, \ldots]$, then the sequence of convergents is $\left\{\frac{0}{1}, \frac{1}{1}, \frac{1}{2}, \frac{3}{5}, \frac{4}{7}, \frac{11}{19}, \frac{15}{26}, \ldots\right\}$. Setting $Q=15$, for instance, we have $k_{4}=5$, $k_{5}=7, j=1 \leq 2=b_{5}$, and $1 \cdot 7+5 \leq Q<2 \cdot 7+5$. Hence the largest (respectively, smallest) rational number with denominator bounded by 15 that is less than (respectively, greater than) $\frac{1}{\sqrt{3}}$ is $\frac{4}{7}$ (respectively, $\frac{1 \cdot 4+3}{1 \cdot 7+5}=\frac{7}{12}$ ), and therefore the two edges in the fundamental arc of $P_{15}$ between whose slopes $\frac{1}{\sqrt{3}}$ lies correspond to the consecutive vectors $(7,4)$ and $(12,7)$ of $V_{15}$.

An irrational number $0<\lambda<1$ is badly approximable if the partial quotients $b_{j}$ in the continued fraction expansion $\lambda=\left[0 ; b_{1}, b_{2}, \ldots\right]$ are bounded, or equivalently if there is a constant $\delta>0$ such that the inequality $\left|\lambda-\frac{a}{q}\right|<\frac{\delta}{q^{2}}$ has no solutions. The set of badly approximable irrationals has Lebesgue measure zero.

Lemma 6. For any irrational number $0<\lambda<1$, we have $\liminf \left(k_{n} / k_{n+1}\right) \leq$ $(\sqrt{5}-1) / 2$.

Proof. Whenever $b_{n} \geq 2$ we have $k_{n} / k_{n+1}=k_{n} /\left(b_{n} k_{n}+k_{n-1}\right)<1 / b_{n} \leq 1 / 2$. Therefore if infinitely many of the $b_{n} \geq 2$, then $\liminf \left(k_{n} / k_{n+1}\right) \leq 1 / 2$. Otherwise, $b_{n}=1$ for $n$ sufficiently large, so the $k_{n}$ eventually satisfy $k_{n+1}=k_{n}+k_{n-1}$. All solutions to this recurrence in positive numbers satisfy $k_{n} \sim c((\sqrt{5}+1) / 2)^{n}$ for some constant $c$; hence $\lim \left(k_{n} / k_{n+1}\right)=((\sqrt{5}+1) / 2)^{-1}=(\sqrt{5}-1) / 2$.

We can now describe the limiting behavior of the local radii of curvature $\tilde{r}_{Q}(\lambda)$.

Theorem 7. Let $0 \leq \lambda \leq 1$.

(a) If $\lambda$ is rational, then $\lim _{Q \rightarrow \infty} \tilde{r}_{Q}\left(\lambda^{+}\right)=\lim _{Q \rightarrow \infty} \tilde{r}_{Q}\left(\lambda^{-}\right)=0$.

(b) If $\lambda$ is irrational, then $\limsup _{Q \rightarrow \infty} \tilde{r}_{Q}(\lambda)$ lies in the interval

$$
\left[\frac{\pi^{2}}{6}\left(1+\lambda^{2}\right)^{3 / 2}, \frac{\pi^{2}}{3}\left(1+\lambda^{2}\right)^{3 / 2}\right] .
$$

(c) $\liminf _{Q \rightarrow \infty} \tilde{r}_{Q}(\lambda)>0$ if and only if $\lambda$ is a badly approximable irrational number.

In particular, for almost all $\lambda$, we have

$$
\limsup _{Q \rightarrow \infty} \tilde{r}_{Q}(\lambda)>0
$$

but

$$
\liminf _{Q \rightarrow \infty} \tilde{r}_{Q}(\lambda)=0
$$

Proof. A straightforward calculation shows that the radius $r$ of the circle passing through the three points $\left(x-x_{1}, y-y_{1}\right),(x, y)$, and $\left(x+x_{2}, y+y_{2}\right)$ satisfies

$$
r^{2}=\frac{1}{4}\left(y_{1}^{2}+x_{1}^{2}\right)\left(y_{2}^{2}+x_{2}^{2}\right)\left(\left(y_{1}+y_{2}\right)^{2}+\left(x_{1}+x_{2}\right)^{2}\right)\left(y_{2} x_{1}-y_{1} x_{2}\right)^{-2} .
$$

To calculate $r_{Q}(\lambda)$, we take $\left(x_{1}, y_{1}\right)=\left(q_{1}, a_{1}\right)$ and $\left(x_{2}, y_{2}\right)=\left(q_{2}, a_{2}\right)$, where $\lambda$ lies between $\frac{a_{1}}{q_{1}}$ and $\frac{a_{2}}{q_{2}}$ in the Farey fractions of order $Q$, and

$$
(x, y)=(X(Q, \lambda), Y(Q, \lambda))
$$


Since $\frac{a_{1}}{q_{1}}$ and $\frac{a_{2}}{q_{2}}$ are consecutive Farey fractions, we know that $a_{2} q_{1}-a_{1} q_{2}=1$, and hence the formula (10) simplifies to

$$
r_{Q}(\lambda)^{2}=\frac{1}{4}\left(a_{1}^{2}+q_{1}^{2}\right)\left(a_{2}^{2}+q_{2}^{2}\right)\left(\left(a_{1}+a_{2}\right)^{2}+\left(q_{1}+q_{2}\right)^{2}\right) .
$$

Now $\frac{a_{1}}{q_{1}}$ and $\frac{a_{2}}{q_{2}}$ are both approximately $\lambda$, so substituting $a_{1} \sim \lambda q_{1}$ and $a_{2} \sim \lambda q_{2}$ into equation (11) and simplifying yields

$$
r_{Q}(\lambda) \sim \frac{1}{2} q_{1} q_{2}\left(q_{1}+q_{2}\right)\left(1+\lambda^{2}\right)^{3 / 2} .
$$

(We record only the main terms for the sake of simplicity. Even a crude estimate such as $\left|\frac{a_{j}}{q_{j}}-\lambda\right|=o\left(\frac{1}{Q}\right)$ would suffice for our purposes.) Therefore

$$
\tilde{r}_{Q}(\lambda)=\frac{r_{Q}(\lambda)}{R(Q)} \sim \frac{q_{1} q_{2}\left(q_{1}+q_{2}\right)}{Q^{3}} \frac{\pi^{2}\left(1+\lambda^{2}\right)^{3 / 2}}{6} .
$$

If $\lambda=\frac{a}{q}$ is a rational number, then in using the expression (12) to calculate $\tilde{r}_{Q}\left(\lambda^{+}\right)$, we would have $q_{1}=q$ for all $Q \geq q$. In particular, since $q_{2} \leq Q$, the numerator grows only quadratically with $Q$, and hence $\lim _{Q \rightarrow \infty} \tilde{r}_{Q}\left(\lambda^{+}\right)=0$. The same argument shows that $\lim _{Q \rightarrow \infty} \tilde{r}_{Q}\left(\lambda^{-}\right)=0$, which proves part (a) of the theorem.

Now suppose that $\lambda=\left[0 ; b_{1}, b_{2}, \ldots\right]$ is irrational. Then there are unique positive integers $n$ and $j \leq b_{n}$ such that $j k_{n}+k_{n-1} \leq Q<(j+1) k_{n}+k_{n-1}$, where the $k_{n}$ are the denominators of the convergents to $\lambda$, in which case $q_{1}=k_{n}$ and $q_{2}=j k_{n}+k_{n-1}$. Thus the expression (12) becomes

$$
\tilde{r}_{Q}(\lambda) \sim \frac{k_{n}\left(j k_{n}+k_{n-1}\right)\left((j+1) k_{n}+k_{n-1}\right)}{Q^{3}} \frac{\pi^{2}\left(1+\lambda^{2}\right)^{3 / 2}}{6} .
$$

In calculating the lim sup of this expression, we should take $Q$ as small as possible, that is, $Q=j k_{n}+k_{n-1}$. If we define $r_{n}=k_{n-1} / k_{n}$, then the expression (13) simplifies to

$$
\tilde{r}_{Q}(\lambda) \sim \frac{k_{n}\left((j+1) k_{n}+k_{n-1}\right)}{\left(j k_{n}+k_{n-1}\right)^{2}} \frac{\pi^{2}\left(1+\lambda^{2}\right)^{3 / 2}}{6}=\frac{j+1+r_{n}}{\left(j+r_{n}\right)^{2}} \frac{\pi^{2}\left(1+\lambda^{2}\right)^{3 / 2}}{6} .
$$

The expression $\frac{j+1+r_{n}}{\left(j+r_{n}\right)^{2}}=\frac{1}{j+r_{n}}+\frac{1}{\left(j+r_{n}\right)^{2}}$ is a decreasing function of both $j$ and $r_{n}$, so in calculating the lim sup it is best to take $j=1$ and $r_{n}$ as small as possible. Therefore

$$
\limsup \tilde{r}_{Q}(\lambda)=\frac{2+\liminf r_{n}}{\left(1+\liminf r_{n}\right)^{2}} \frac{\pi^{2}\left(1+\lambda^{2}\right)^{3 / 2}}{6},
$$

and by Lemma 6 the first fraction lies in the interval $\left[\frac{2+(\sqrt{5}-1) / 2}{(1+(\sqrt{5}-1) / 2)^{2}}, \frac{2+0}{(1+0)^{2}}\right]=[1,2]$. This establishes part (b) of the theorem.

Similarly, in calculating the lim sup of the expression (13), we should take $Q$ as large as possible, that is, $Q=\left((j+1) k_{n}+k_{n-1}\right)^{-}$, in which case (13) simplifies to

$$
\tilde{r}_{Q}(\lambda) \sim \frac{k_{n}\left(j k_{n}+k_{n-1}\right)}{\left((j+1) k_{n}+k_{n-1}\right)^{2}} \frac{\pi^{2}\left(1+\lambda^{2}\right)^{3 / 2}}{6}=\frac{j+r_{n}}{\left(j+1+r_{n}\right)^{2}} \frac{\pi^{2}\left(1+\lambda^{2}\right)^{3 / 2}}{6} .
$$

The expression $\frac{j+r_{n}}{\left(j+1+r_{n}\right)^{2}}$ always lies between $\frac{j+1}{(j+2)^{2}}$ and $\frac{j}{(j+1)^{2}}<\frac{1}{j}$, which are decreasing functions of $j$, so in calculating the lim inf it is best to take $j$ as large as possible, that is, $j=b_{n}$. If $\lambda$ is badly approximable, so that $b_{n} \leq B$ for all $n$, then

$$
\liminf \tilde{r}_{Q}(\lambda) \geq \frac{B+1}{(B+2)^{2}} \frac{\pi^{2}\left(1+\lambda^{2}\right)^{3 / 2}}{6}>0 ;
$$


if $\lambda$ is not badly approximable, then the $b_{n}$ are unbounded above, and hence

$$
\liminf \tilde{r}_{Q}(\lambda) \leq \liminf \frac{1}{b_{n}} \frac{\pi^{2}\left(1+\lambda^{2}\right)^{3 / 2}}{6}=0 .
$$

This establishes part (c) of the theorem.

The two examples in Figure 6 illustrate the two possibilities in part (c) of the theorem. As noted before, the continued fraction expansion of $\frac{1}{\sqrt{3}}$ is $[0 ; 1,1,2,1,2,1$, $2, \ldots]$, and so in particular $\frac{1}{\sqrt{3}}$ is badly approximable, since the partial quotients are bounded above by 2 . We can see the repeating groups of a single curve followed by a pair of curves in the left-hand graph; in particular, the near-periodicity of the graph implies that the values of $\tilde{r}_{Q}\left(\frac{1}{\sqrt{3}}\right)$ are bounded below. On the other hand, the continued fraction expansion of $e-2$ is $[0 ; 1,2,1,1,4,1,1,6,1,1,8, \ldots]$, and in particular $e-2$ is not badly approximable since the partial quotients are unbounded. In the right-hand graph we can see the influence of these partial quotients (the last full group contains two single curves and a group of 14 curves, corresponding to the string 1,1,14 in the continued fraction), and in particular that the lim inf of the values of $\tilde{r}_{Q}(e-2)$ is zero.

We remark that the possible values for $\lim \inf \left(k_{n} / k_{n+1}\right)$ in Lemma 6 are closely related to the Markov spectrum. Thus the possible values for $\lim \sup _{Q \rightarrow \infty} \tilde{r}_{Q}(\lambda)$, as well as the possible values for $\liminf _{Q \rightarrow \infty} \tilde{r}_{Q}(\lambda)$ for badly approximable irrationals $\lambda$, are also related to the Markov spectrum.

\section{ACKNOWLEDGEMENTS}

The author acknowledges the support of the Department of Mathematics of the University of British Columbia and of the Natural Sciences and Engineering Research Council. The author also thanks Bill Casselman for pointing out the irregularity of the local curvatures and for contributing the graphics in Figures 1 and 2 ,

\section{REFERENCES}

1. M. N. Huxley, Area, lattice points, and exponential sums, The Clarendon Press, Oxford University Press, New York, 1996, Oxford Science Publications. MR 97g:11088

2. Alex Iosevich, Curvature, combinatorics, and the Fourier transform, Notices Amer. Math. Soc. 48 (2001), no. 6, 577-583. MR 2002e:42004

3. V. Jarník, Über die Gitterpunkte auf konvexen Kurven, Math. Zeitschrift 24 (1925), 500-518.

4. Ivan Niven, Herbert S. Zuckerman, and Hugh L. Montgomery, An introduction to the theory of numbers, fifth ed., John Wiley \& Sons Inc., New York, 1991. MR 91i:11001

5. A. M. Vershik, The limit form of convex integral polygons and related problems, Funktsional. Anal. i Prilozhen. 28 (1994), no. 1, 16-25, 95; English transl., Functional Anal. Appl. 28 (1994), 13-20. MR 95i:52010

Department of Mathematics, University of British Columbia, Room 121, 1984 Mathematics Road, Vancouver, British Columbia, Canada V6T $1 Z 2$

E-mail address: gerg@math.ubc.ca 